\title{
Severe Unresolved Cholestasis Due to Unknown Etiology Leading to Early Allograft Failure Within the First 3 Months of Liver Transplantation
}

\author{
Roberta Angelico, MD, ${ }^{1,2}$ Undine A. Gerlach, MD, ${ }^{1,3}$ Bridget K. Gunson, ${ }^{1,4}$ Desley Neil, MD, ${ }^{5}$ \\ Hynek Mergental, MD, ${ }^{1,4}$ John Isaac, FRCS, ${ }^{1}$ Paolo Muiesan, FRCS, ${ }^{1}$ Darius Mirza, FRCS, ${ }^{1,4}$ \\ and $M$. Thamara PR Perera, FRCS ${ }^{1}$
}

\begin{abstract}
Background. Causes of severe cholestasis after liver transplantation (LT) are multi-factorial. Although the etiology is predictable in some, others culminate in graft/patient loss without a definitive cause identified. Severe cholestasis is usually associated with overlapped histological findings of rejection and biliary features, and diagnostic interpretation may pose a challenge. Methods. This is 10-year retrospective analysis of patients with unexplained severe cholestasis resulting in death/graft loss within 90 days of LT. Of $1583 \mathrm{LT}$ during the study period, 90-day graft failure occurred in 129 (8\%) cases; a total of 45 (3\%) patients had unresolving severe cholestasis (bilirubin, $>100 \mathrm{\mu mol} / \mathrm{L}$; alkaline phosphatase, $>400 \mathrm{UI} / \mathrm{L}$ after 15 days from $\mathrm{LT}$ ), excluding those due to primary nonfunction/sepsis/vascular causes $(n=84)$. Demographics, allograft biopsies, radiological investigations, and clinical outcome were analyzed. Results. All patients had persistent abnormal liver biochemistry. Doppler ultrasound scan was normal in all cases. Thirty-five (78\%) recipients had at least 1 allograft biopsy (2 [1-9]). On the first biopsy, 22 (63\%) patients had acute rejection, 4 (18\%) early-chronic rejection, 12 (34\%) antibody-mediated rejection. In subsequent biopsies chronic rejection was evident in $5(14 \%)$ cases. Donor-specific antibodies were detected in all patients tested. Biliary anatomy was studied in detail in 9 (20\%) patients, all presenting biliary strictures. The majority $(n=39 ; 87 \%)$ died within 32 (10-91) days, only survivors were from retransplantation $(n=3 ; 6.5 \%)$ and biliary intervention $(n=3 ; 6.5 \%)$. Conclusions. Unresolving severe cholestasis after $L T$ is a key parameter predicting patient/allograft outcome. Histologically, rejection seems to overlap with biliary strictures; hence, allograft biopsy with signs of rejection should not be a reason to overlook biliary problems, in particular when biliary features are present. Only extensive radiological investigation/intervention or retransplantation prevents patient/allograft loss.
\end{abstract}

(Transplantation 2018;102: 1307-1315)

holestasis is not uncommon after liver transplantation (LT) and well recognized etiologies include anastomotic or nonanastomotic biliary strictures, early allograft dysfunction (EAD), severe sepsis and viral hepatitis such as early hepatitis $\mathrm{C}$ recurrence or cytomegalovirus hepatitis or even small for size syndrome. ${ }^{1,2}$ In addition, graft rejection may present as cholestasis; some cases of acute cellular rejection (ACR) progress to severe cholestatic and ductopenic

Received 25 June 2017. Revision received 18 January 2018.

Accepted 23 January 2018.

${ }^{1}$ The Liver Unit, Queen Elizabeth Hospital Birmingham, Birmingham, United Kingdom. ${ }^{2}$ Department of Abdominal Transplantation and Hepatobiliary and Pancreatic Surgery, Bambino Gesù Children's Hospital IRCCS, Rome, Italy.

${ }^{3}$ Department of General, Visceral and Transplantation Surgery, Charité Universitätsmedizin Berlin, Campus Virchow Klinikum, Berlin, Germany.

${ }^{4}$ NIHR Birmingham Biomedical Research Centre at the University Hospitals Birmingham and the University of Birmingham, Birmingham, United Kingdom.

${ }^{5}$ Histopathology Unit, Queen Elizabeth Hospital Birmingham, Birmingham, United Kingdom.

B.K.G., H.M., and D.F.M. are supported by the NIHR Birmingham Biomedical Research Centre.

The authors declare no conflicts of interest. chronic rejection within a very short period despite institution of salvage immunosuppression therapy. ${ }^{3}$ Also antibodymediated rejection (AMR) may have cholestatic biochemistry and biliary features on biopsy. ${ }^{4-6}$

Early graft loss within first 3 months after LT is a problem faced by the transplant community. This may be sporadic in low volume centers, but in high volume centers several cases may be encountered each year and the reported incidence

R.A. participated in the data collection, analysis and interpretation, and wrote the article. U.A.G. participated in the data collection, analysis and interpretation, and wrote the article. B.G. participated in the data collection, analysis, and interpretation. D.N. participated in the data collection, review of allograft biopsies, analysis, and intellectual content. H.M. participated in the data collection, analysis, and interpretation. J.R.I. participated in the data collection, analysis and interpretation, intellectual content. P.M. participated in the data collection, analysis and interpretation, and intellectual content. D.F.M. conceptualized the study, data interpretation, and intellectual content. M.T.P.R.P. designed the study, data analysis and interpretation, intellectual content.

Correspondence: Thamara Perera, FRCS, Liver Unit, Queen Elizabeth hospital Birmingham, Edgbaston, Birmingham B15 2TH, United Kingdom. (Thamara.Perera@uhb.nhs.uk).

Copyright @ 2018 Wolters Kluwer Health, Inc. All rights reserved.

ISSN: 0041-1337/18/10208-1307

DOI: $10.1097 /$ TP.0000000000002139

Transplantation august 2018 - Volume 102 Number 8

www.transplantjournal.com 
may even reach up to $5 \%$ of all transplant cases. Early allograft loss has a considerable added burden to transplant programs. The problem lies with difficulty in diagnosing the exact etiology. Histology may show overlapping biliary and rejection features, which may also overlap in etiology: the biliary features may be secondary to the rejection but obstruction cannot be excluded histologically and it is increasingly recognized that biliary obstruction may occur as a consequence of AMR.

Isolated severe cholestasis should be considered as a different clinical entity from traditionally described "delayed graft function" (DGF), also termed as "early allograft dysfunction" (EAD), ${ }^{7-10}$ where severe early hepatocyte injury has been identified as the major cause of graft dysfunction. EAD is often secondary to a combination of donor and recipient characteristics ${ }^{11}$ as well as surgical factors, such as length of total ischemia ${ }^{12}$ and ischemia/reperfusion injury, ${ }^{13}$ requiring often organ support before complete restitution of normal graft functions or succumbing to sequelae such as severe sepsis.

LT recipients with severe cholestasis in the present study are an entirely different cohort than those fulfilling the criteria for EAD. This group of patients recovers well within the early postoperative period and is fit enough to be transferred to the transplant ward from the intensive care unit, thus not requiring prolonged organ support and is free of sepsis, and presenting asymptomatic elevation of liver function tests (LFTs). Routine imaging usually rules out vascular causes and they often undergo liver biopsy.

Through this study we aim to resolve this diagnostic dilemma through retrospective review of the histology report of all LT recipients who developed unexplained severe cholestasis within 3 months in a large volume transplant center in Europe, with the aim to define causes and pitfalls in their management.

\section{MATERIALS AND METHODS}

\section{Patient Population and Clinical Data}

This is a retrospective review of all LT performed between January 2004 and January 2014 at The Liver Unit, Queen Elizabeth Hospital Birmingham, United Kingdom. All recipients with persistent severe cholestasis (defined as persistent bilirubin $>100 \mu \mathrm{mol} / \mathrm{L}$ and alkaline phosphatase $[\mathrm{ALP}]>400 \mathrm{UI} / \mathrm{L}$ after 15 days from LT) and graft failure within 3 months from LT were included in the study. LT recipients with a definitive identifiable etiology or complication leading to graft failure such as primary nonfunction (PNF), hepatic artery thrombosis (HAT), other vascular complications (venous occlusion/portal venous thrombosis) and sepsis (proved by positive microbiology) were excluded. Clinical data were collated from the electronic clinical database including donor and recipient demographic characteristics, intra-operative details, postoperative LFTs, radiological investigation, treatment for graft rejection and postoperative clinical outcome and pathology report. The Donor Risk Index was calculated using the formula defined by Feng at al. ${ }^{14}$ This study was approved by the clinical audit and research management system (CARMS) of the institution (registration number: CARMS-11425).

\section{Histological Data}

Protocol time zero biopsy was defined as a biopsy taken in the immediate postreperfusion period, and comparisons were made when this information was present. Posttransplant biopsies were performed at varied time points to investigate the cause of the cholestasis. All liver biopsies were reported by experienced LT histopathologists at the time of diagnostic biopsy and were re-reviewed by 1 histopathologists to look for features of AMR, ${ }^{4}$ not defined in the earlier part of the study.

The histological features assessed were those of (1) ACR (portal infiltrate, bile duct inflammation and damage, venous inflammation and central perivenulitis), ${ }^{15}$ (2) early chronic rejection (paucity of bile ducts and "dysplastic/sick" bile ducts), ${ }^{15,16}$ (3) AMR (portal oedema, ductular reaction, portal microvasculitis, sinusoidal infiltrate, arteritis and eosinophilic venulitis). ${ }^{15,17,18}$ The rejection activity index (RAI) was determined. The severity of ACR was classified as indeterminate, mild, moderate or severe according to standard criteria. ${ }^{15}$ The severity of bilirubinostasis/cholestasis was also assessed. Donor-specific antibody (DSA) testing was not performed routinely, but in recent years, DSA testing has been performed in cases of histological or clinical suspicion. Hepatic steatosis was defined as microvesicular or macrovesicular steatosis and classified into mild $(<30 \%)$, moderate $(30-60 \%)$ or severe $(>60 \%)$. $^{19}$

\section{Statistical Analysis}

Data were obtained from a prospectively collected consecutive database (Infoflex; Chameleon Information Management Systems). Donor/recipient characteristics and clinical data are shown (wherever applicable) as either median with range or mean \pm standard deviation. Univariate data were analyzed using the Mann-Whitney test and Fisher exact test. A $P$ value less than 0.05 was considered significant. Normal distribution continuous data were analyzed by parametric test (Student $t$ test). Survival rates were calculated using the Kaplan-Meier method for univariate analysis and Cox regression for multivariate analysis. The program used for statistical analysis was SPSS 13.0 (233 South Wacker Drive, Chicago, IL) for Windows.

\section{RESULTS}

\section{Donor, Recipient, and Transplant Characteristics}

A total of 1583 LT were performed during the study period. One hundred twenty-nine $(8 \%)$ recipients had graft

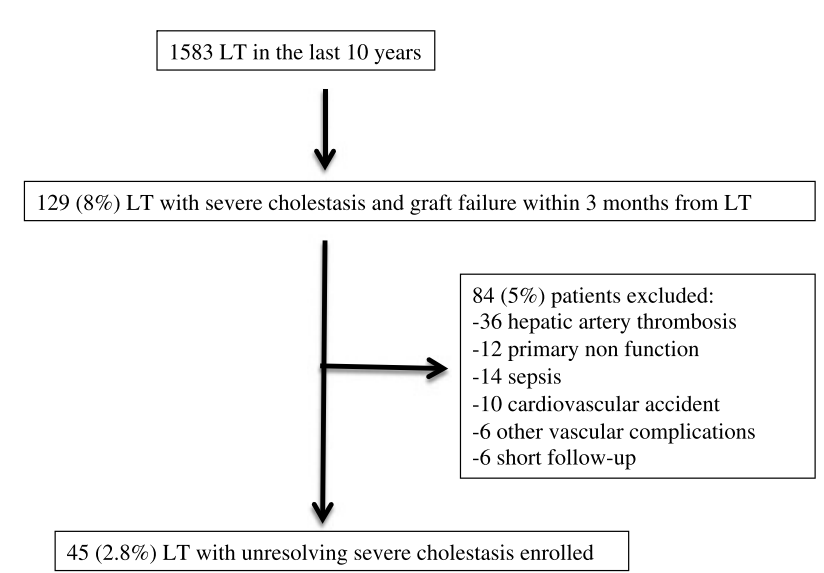

FIGURE 1. Study population selection for unresolving severe cholestasis after LT. All patients undergoing LT in the last 10 years at our institution, who developed graft failure within 3 months from LT, associated with unresolving severe cholestasis and without a definitive cause, were included. Severe cholestasis was defined as persistent bilirubin > $100 \mu \mathrm{mol} / \mathrm{L}$ or ALP > $400 \mathrm{UI} / \mathrm{L}$ after 15 days from LT. 
TABLE 1.

Recipient, donor, and surgical characteristics of the study population

\begin{tabular}{|c|c|}
\hline Recipient variables & Number (range) \\
\hline No. patients & 45 \\
\hline Sex (male/female) & $33 / 12$ \\
\hline Age, y & 49 (17-71) \\
\hline MELD score & $18(8.0-35.0)$ \\
\hline UKELD score & $54(45.0-68.0)$ \\
\hline \multicolumn{2}{|l|}{ Indication for LT } \\
\hline Primary sclerosing cholangitis & $3(7 \%)$ \\
\hline Alcoholic cirrhosis & $12(27 \%)$ (5 with HCC) \\
\hline HCV-related cirrhosis & 8 (18\%) (1 with HCC) \\
\hline HBV-related cirrhosis & 1 (2\%) (1 with HCC) \\
\hline Primary biliary cirrhosis & $4(9 \%)$ \\
\hline Secondary biliary cirrhosis & $1(2 \%)$ \\
\hline Regraft & 6 (14\%) (4 PNF, 2 HAT) \\
\hline Autoimmune hepatitis & $2(4 \%)$ \\
\hline Seronegative hepatitis & $1(2 \%)$ \\
\hline Caroli disease & $1(2 \%)$ \\
\hline Cryptogenic cirrhosis & $1(2 \%)$ \\
\hline Amyloidosis & $1(2 \%)$ \\
\hline NASH & $1(2 \%)$ \\
\hline Acute liver failure & $3(2 \%)$ \\
\hline \multicolumn{2}{|l|}{ Comorbidities } \\
\hline Circulatory disease & $6(13 \%)$ \\
\hline Diabetes type II & $6(13 \%)$ \\
\hline Renal impairment (not dialysis) & $4(9 \%)$ \\
\hline Asthma & $4(9 \%)$ \\
\hline Ulcerative colitis & $2(4 \%)$ \\
\hline Cystic fibrosis & $1(2 \%)$ \\
\hline \multicolumn{2}{|l|}{ Donor variables } \\
\hline Donor age, y & $46(18-69)$ \\
\hline Donor graft type (DBD/DCD) & $39 / 6$ \\
\hline $\mathrm{DR}$ & $1.7(1.04-2.9)$ \\
\hline \multicolumn{2}{|l|}{ Surgical variables } \\
\hline Duration of surgery (range), $\mathrm{h}$ & $5(3-8)$ \\
\hline CIT (range), min & $492(166-768)$ \\
\hline WIT (range), min & $41(29-68)$ \\
\hline Whole graft/split graft & $38 / 7$ \\
\hline Biliary anastomosis (duct-to-duct/HJ) & $38 / 7$ \\
\hline ITU stay, d & $21(1-49)$ \\
\hline
\end{tabular}

Values reported as medians and ranges.

$\mathrm{DBD}$, donor after brain death; DCD, donor after circulatory death; DRI, donor risk index; MELD, Model for End-Stage Liver Disease; UKELD, United Kingdom Model for End-Stage Liver Disease; HCC, hepatocellular carcinoma; HCV, hepatitis C virus; HBV, hepatitis B virus; NASH, nonalcoholic steatohepatitis; CIT, cold ischemic time; WIT, warm ischemic time; HJ, hepaticojejunostomy; ITU, intensive therapy unit.

failure associated with persistent severe cholestasis (as defined above) within 3 months from LT. Of these, 84 (5\%) patients with known causes of graft failure, including HAT $(\mathrm{n}=36 ; 2 \%), \operatorname{PNF}(\mathrm{n}=12 ; 0.7 \%)$, definitive diagnosis of sepsis $(\mathrm{n}=14 ; 0.9 \%)$, postoperative cardiovascular accident $(\mathrm{n}=10 ; 0.6 \%)$, other vascular complications $(\mathrm{n}=6 ; 0.4 \%)$ and short postoperative follow-up $([6 ; 0.4 \%]$, median, 7 days [3-11]) were excluded (Figure 1).

This resulted in 45 (3\%) LT recipients (male/female, 33/12; median age, 49 [17.4-70.5] years) with severe cholestasis of unknown cause leading to graft loss within a median time of 32 days (15-91 days) from LT included in this study (Table 1). During the study period, the average number of patients with cholestasis of unknown etiology was 4 cases per year (range, $2-6$ cases). Eight (18\%) recipients had a split LT, and $1(2 \%)$ patient received a combined liver-kidney transplant. Biliary anastomosis was performed as duct-toduct biliary reconstruction in $38(84 \%)$ cases, whereas 7 (16\%) recipients had Roux-en Y choledochojejunostomy. All split recipients had a T-tube kept in place up to 3 months, as is local practice.

Thirty-nine $(87 \%)$ patients received a graft from a donor after brain death and $6(13 \%)$ patients from a donor after circulatory death. Recipient, donor, and surgical variables are reported in Table 1.

The baseline variables of the study population were compared with 1538 adult LT recipients who did not experience unresolved cholestasis, transplanted in the same period at our institution. The study cohort showed similar baseline characteristics to these of LT recipients without unresolved cholestasis including recipient age (49 [17.4-70.5] years vs 52 [16.4-73.4] years, $P=$ n.s.), Model for End-Stage Liver Disease at the time of LT (18 [8.0-35.0] vs 17 [6-40], $P=$ n.s.), donor age (46 [18-69] years vs 48 [6-83] years; $P=$ n.s.), whereas only the recipient sex was different in the 2 groups (male, $\mathrm{n}=33[73.3 \%]$ vs $\mathrm{n}=882[57.3 \%], P=0.043$ ).

\section{Biochemical Findings and Immunosuppression Trough Levels}

The median LFT levels during the observational period are reported in Figure 2. The bilirubin level increased from $88.5(19-317) \mu \mathrm{mol} / \mathrm{L}$ on day 1 to 135 (101-573) $\mu \mathrm{mol} / \mathrm{L}$ on day $7(P=0.003)$. At 15, 30, 60, and 90 days after LT, bilirubin levels were $170(102-573) \mu \mathrm{mol} / \mathrm{L}, 239$ (58-615) $\mu \mathrm{mol} / \mathrm{L}$,
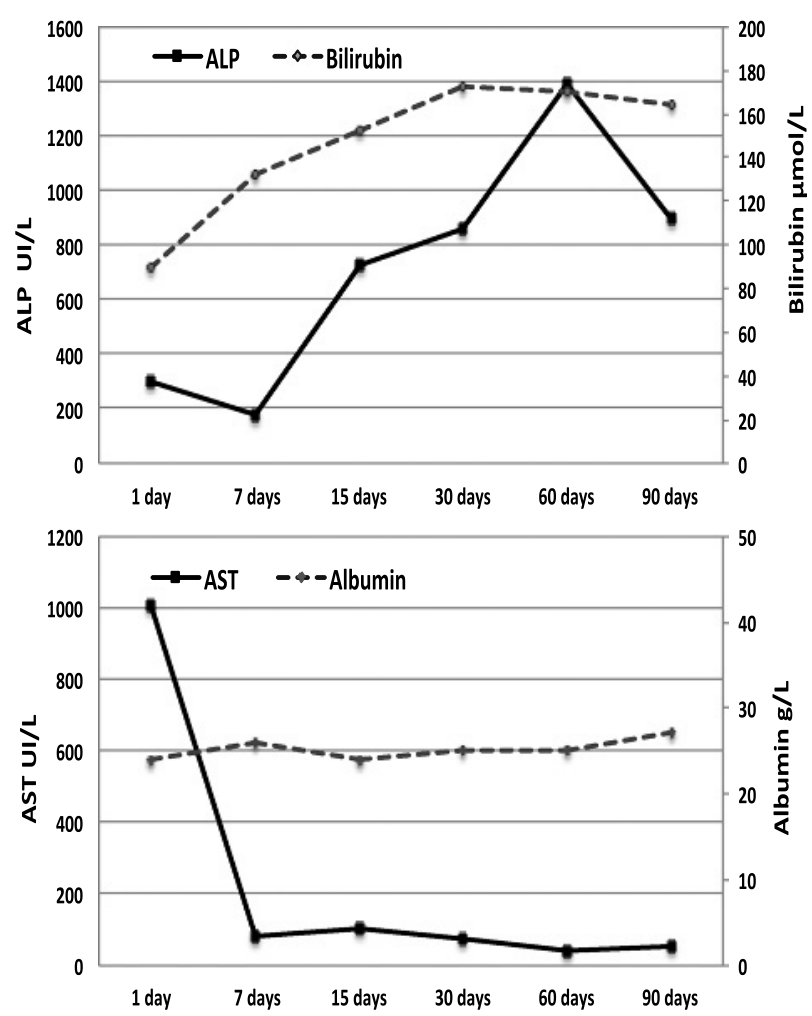

FIGURE 2. The biochemical course within 3 months from LT. Values reported as medians. ALP, UI/L; AST, UI/L; bilirubin, $\mathrm{mmol} / \mathrm{L}$; albumin, g/L. 
$245 \quad(45-706) \mu \mathrm{mol} / \mathrm{L}$, and $115 \quad(69-250) \mu \mathrm{mol} / \mathrm{L}$, respectively $(P=$ n.s. $)$.

All patients received the local standard immunosuppression regimen, comprising: prednisolone $20 \mathrm{mg}$ daily (gradually reduced by $5 \mathrm{mg} /$ day every 3 weeks and stopped after 12 weeks), Azathioprine (1-2 mg/kg per day) or mychophenolate ( $1 \mathrm{~g}$ twice/day), Tacrolimus $(0.1 \mathrm{mg} / \mathrm{kg}$ per day). At $7,15,30$, 60 , and 90 days after LT, the tacrolimus trough levels were 9.1 (1.1-17.1) ng/mL, 7.05 (1.1-16.2) ng/mL, 8.01 (1.112.1) ng/mL, 8.15 (1.1-14.1) ng/mL, and 6.7 (1.1-12.6) mg/mL, respectively $(P=$ n.s. $)$. No significant variations in tacrolimus blood level were observed during follow-up.

\section{Radiological Investigations}

During the postoperative period, the median number of Doppler ultrasound (US) scans for each patient was 5 (range, 2-14), which in all cases were negative for intrahepatic biliary dilatation and vascular complications. Twenty-six $(58 \%)$ patients had a computed tomography scan, and in 9 cases, an abdominal collection amenable to percutaneous drainage was identified. Biliary anatomy was studied in detail in 9 $(20 \%)$ cases by magnetic resonance cholangiopancreatography (MRCP, [n = 5]), endoscopic retrograde cholangiopancreatography (ERCP) $(n=7)$ and/or percutaneous transhepatic cholangiography (PTC) $(n=4)$. All specific biliary imaging performed suggested that the cause of persistent cholestasis was of biliary origin as in mechanical obstruction; a total of $9(100 \%)$ cases reported biliary strictures, which were associated with anastomotic bile leak in 3 cases (all of them received a split graft). Of 5 patients who underwent MRCP, 2 patients were treated with PTC biliary stent, 2 cases had combined PTC and ERCP biliary stenting, whereas 1 case had conservative management. Endoscopic retrograde cholangiopancreatography was performed in another 5 patients, but a biliary stent was placed in only in 3 of them. Of 7 LT recipients who received a biliary interventional treatment, the biochemical cholestasis resolved in only 3 cases.

\section{Histological Findings}

Thirty-four $(76 \%)$ recipients had a time zero liver biopsy, graft reperfusion injury was observed in $30(88 \%)$ biopsies (20 mild, 9 moderate, 1 severe), and features of graft steatosis were reported in $20(59 \%)$ cases (19 mild, 1 moderate).

After LT, $35(78 \%)$ recipients underwent at least 1 allograft biopsy as an investigative tool for persistent cholestasis. The median number of biopsies per patient was 2 (range, 1-9) (20 having 1 biopsy, 5 having 2 biopsies, 3 having 3 biopsies, 3 having 4 biopsies [ 1 also with an explant], 1 having 5

\section{TABLE 2.}

Major histological findings at the graft biopsies after LT

\begin{tabular}{lc}
\hline Histological findings & Number (range, \%) \\
\hline Recipients underwent graft biopsy & $35(78 \%)$ \\
Graft biopsies for patient & $2(1-9)$ \\
ACR & $22(63 \%)$ \\
$\quad$ Mild & 8 \\
$\quad$ Moderate & 7 \\
Severe & 7 \\
AMR & $12(34 \%)$ \\
Early chronic rejection & $4(18 \%)$ \\
Chronic rejection & $5(14 \%)$ \\
\hline
\end{tabular}

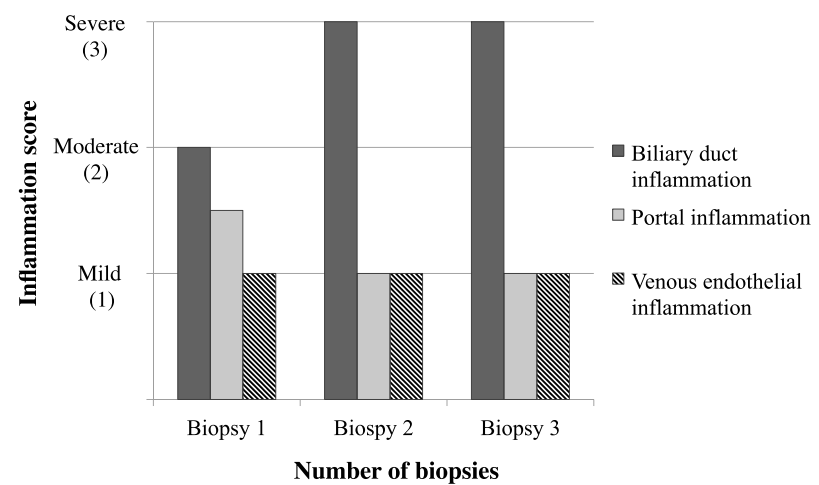

FIGURE 3. Severity of cellular rejection components in the allograft biopsies after LT. The RAI was used to score liver allograft biopsies with acute rejection. The RAl score was defined by the presence of portal inflammation (score, 1-3), venous endothelial inflammation (score, 1-3) and biliary duct inflammation (score, 1-3) at biopsy numbers 1, 2, and 3 performed during the observational period. Values are reported as medians.

biopsies and 1 having 9 biopsies and graft explant during the follow-up period). Histological findings are summarized in Table 2.

On the first biopsy, $22(63 \%)$ of 35 patients had ACR $(8$ mild, 7 moderate, 7 severe), whereas 2 cases were indeterminate for rejection. The median RAI score was $4(0-9)$ with histological inflammations score (portal 1.5 [0-3], bile duct 2 [0-3], venous endothelial 1 [0-3]) (Figure 3). Four biopsies had features of early chronic rejection. Twelve $(34 \%)$ biopsies had features suspicious for AMR $(5$ highly suspicious), all but 1 with concurrent ACR, the other developed ACR in subsequent biopsies. Two patients had a donor-derived steatohepatitis.

Over subsequent biopsies, chronic rejection was developed in $5(14 \%)$ patients, and 1 patient showed venous outflow obstruction. Histological features suspicious of AMR became more striking in 1 patient but did not develop in any when not present in the first biopsy, with the exception of finding a fibrinoid arteritis in conjunction with a foam cell arteriopathy in the explant of 1 patient transplanted for chronic rejection. C4d staining was done in at least 1 biopsy of 12 patients and was positive in 4 patients.

Three of those highly suspicious for AMR did not have a C4d stain and 3 had a variably positive C4d stain by immunoperoxidase. Of the 3 more recent cases in which the C4d stain had been performed, all had a DSA detected. Four of the 5 slightly suspicious for AMR cases did not have a C4d stain, whereas 1 was negative by immunoperoxidase. The patients with established chronic rejection did not have histological features suspicious for AMR. Bilirubinostasis (24 severe, 8 moderate, 2 mild) were present in all biopsies (Figure 4).

Twelve $(27 \%)$ patients with persistent abnormal LFTs underwent second and third biopsies, and in 9 cases, features of ACR ( 4 mild, 4 moderate, 4 severe) persisted, and in 2 cases, features evolved to chronic rejection, and DSA were detected in both cases.

\section{Clinical Outcome and Survival}

Postoperative complications included renal impairment $(\mathrm{n}=31$ [69\%], of which 2 cases [4\%] required long-term dialysis), gastric bleeding $(\mathrm{n}=1)$, bowel perforation $(\mathrm{n}=1)$, and 


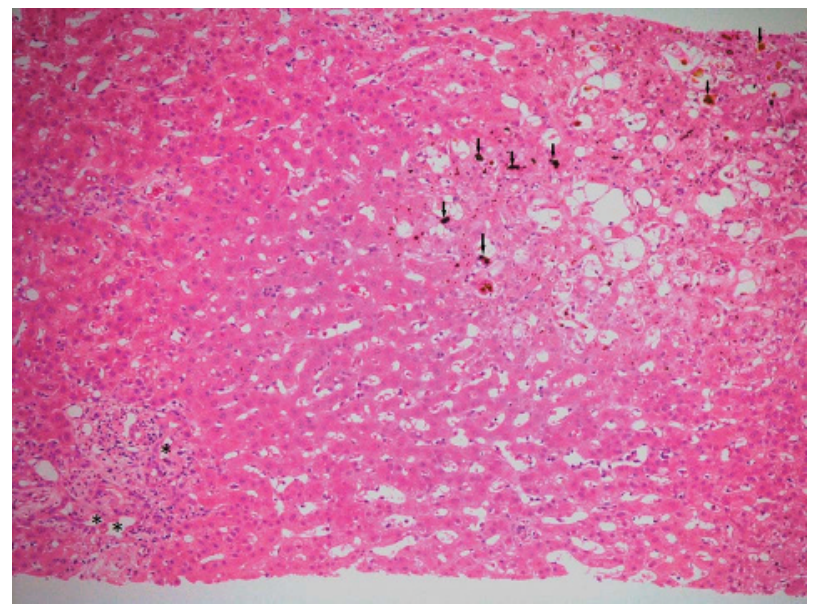

FIGURE 4. Histological features of cholestasis and acute rejection in the early period after LT. Hematoxylin and eosin-stained section showing a portal tract in the bottom left with dilated bile ductules (*) and mild portal inflammation. There is severe bilirubinostasis with numerous bile plugs, predominantly in a centrilobular position (arrows) (original magnification, $\times 10$ ).

enteric leak $(n=1)$ requiring second surgery for bowel resection; cerebrovascular accident $(\mathrm{n}=1)$.

Twenty-six (74\%) patients were treated for ACR with high doses of steroids (prednisolone $200 \mathrm{mg}$ for 3 days), and the median number of treatments was 2 (1-4). All patients with biliary strictures $(n=9)$ had previously histological prove of ACR and severe cholestasis. In particular, 7 recipients had a first liver biopsy showing mild $(\mathrm{n}=4)$ and moderate $(\mathrm{n}=3)$ ACR and were treated with standard doses of steroid boluses without resolution of cholestasis; the second liver biopsy did not show any signs of rejection (but persistent severe cholestasis), thus later, they underwent biliary anatomy investigations and were treated with biliary stents $(n=5)$ or conservative management $(\mathrm{n}=2)$. The remaining 2 patients were treated with steroids boluses twice for ACR (after histological proven in 2 consecutive graft biopsies); however, the biochemical cholestasis was not resolved, therefore, both underwent ERCP with biliary stent. Despite failure to respond to antirejection treatment and/or ongoing cholestasis without obvious cause, 17 of the remaining patients were not specifically investigated for a biliary cause.

The majority of recipients $(\mathrm{n}=39 ; 87 \%)$ died within 32 $(10-91)$ days. The only survivors $(n=6 ; 13 \%)$ were $3(7 \%)$ patients who underwent retransplantation ( 2 for chronic rejection and 1 for acute on chronic AMR) and $3(7 \%)$ recipients who underwent intervention for biliary strictures. The diagnostic and therapeutic management and patient outcomes are summarized in Figure 5. On univariate and multivariate analyses, there was no significant association of all other variables with patient survival. The survival rate at 3 months in this group, therefore, from LT was $13 \%$ (Figure 6).

From a clinical point of view, the primary cause of graft loss was defined as rejection (histologically proven) in 21 patients, biliary complications in 5 cases (of which 3 had a proven DSA and features of AMR on their biopsies before the development of the stricture) and unknown causes in 10 $(26 \%)$ recipients, of which 2 had donor derived steatohepatitis. Unfortunately, testing for DSAs and C4d staining was not performed in the majority of these patients. During the

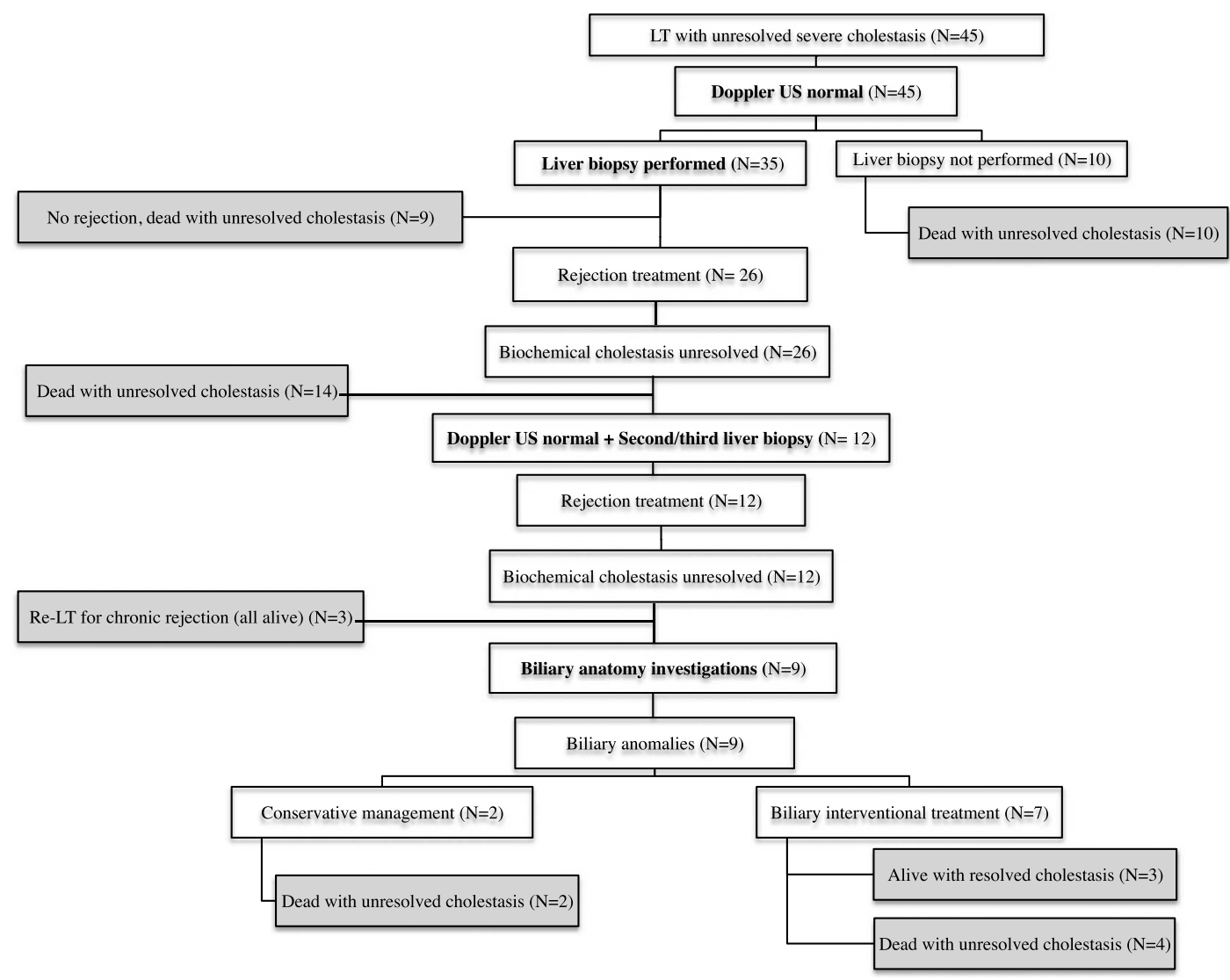

FIGURE 5. Diagnostic and therapeutic management and patient outcomes. N, number; re-LT, re-LT. 


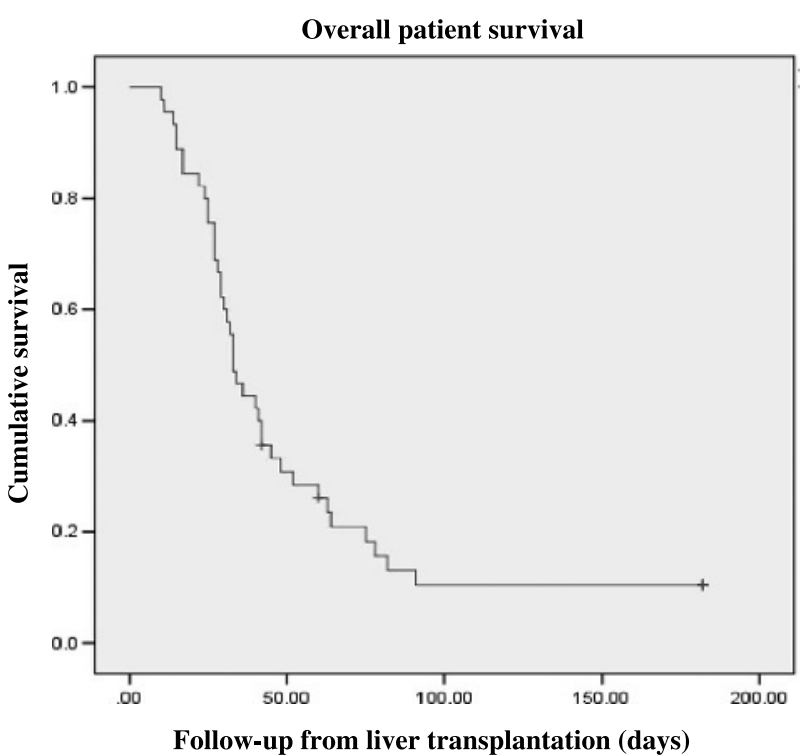

FIGURE 6. Survival rate after 3 months from LT of recipients who developed severe unresolving cholestasis.

observational period, the overall yearly graft dysfunction rate was $4.5 \%$.

Overall rejection (histologically proven) may have been the predominant cause $(60 \%)$, whereas rejection was not likely to have contributed in $14(40 \%)$ patients.

In the 3 LT patients who underwent retransplantation, the liver grafts explanted at the time of the second transplant were analyzed; other patients did not have postmortem liver data. Of the 3 retransplanted patients, 1 underwent a second transplantation for chronic rejection, the diagnosis of which was confirmed on the explanted liver in association with severe ductopenia (C4d negative); the explant graft of the second patient who was retransplanted for chronic rejection showed features of chronic rejection, moderate ACR, severe centrilobular bilirubinostasis and focal microvesicular and macrovesicular steatosis. In the third patient, retransplanted for acute on chronic AMR, the histology of the explant graft showed signs of early cirrhosis and concomitant venoocclusive lesions secondary to either AMR (Cd4 positive) or ACR.

\section{DISCUSSION}

Severe cholestasis is a frequent event early after LT as a consequence of a number of disparate disease processes with different mechanisms. In the immediate posttransplant period, severe intrahepatic cholestasis is mainly related to preservation reperfusion injury, which may cause damage to the bile canaliculi and is the major reason for EAD, contributing to a greater than $20 \%$ mortality rate after $\mathrm{LT}^{20}$ Other causes, which may present with cholestasis in the first weeks after LT, include vascular complications such as HAT and venous outflow obstruction, biliary anastomotic stricture or leak, and ACR. ${ }^{1}$ Intrahepatic cholestasis may also be due to extrahepatic conditions such as sepsis, ${ }^{21}$ cytomegalovirus infection, ${ }^{22}$ and drugs, for example, cyclosporine. ${ }^{23}$

Transplant surgeons and physicians are routinely dealing with the management of such conditions to identify the major causes of cholestasis. Commonly, the initial diagnostic approach is by Doppler US followed by allograft percutaneous biopsy, when required. This pathway is considered sufficient to exclude the most common cause; however, a small proportion of recipients may develop persistent severe cholestasis during the first weeks after LT without apparent definitive cause detected by these routine investigations.

Doppler US is the most economical and cost-effective imaging modality for evaluating postoperative fluid collections, vascular and biliary complications after LT. Usually, when bile duct dilatation, stones and/or leakage are identified by US, patients are referred for therapeutic ERCP or PTC..$^{24-26}$ In contrast, in cases with a normal abdominal US and persistent deranged LFTs, a liver biopsy is commonly performed to exclude rejection. The interpretation however is not always straightforward with a broad spectrum of pathological processes seen early posttransplant and often there is more than 1 pathological process occurring. During the majority of this study period, the histological features of AMR were not described and hence not looked for and the clinical relevance is only now starting to be appreciated. ${ }^{15,17,18}$

In the first few weeks after LT, ACR (often associated with superimposed changes secondary to preservation-reperfusion injury) is the most common histological findings. ${ }^{9,27}$ Moreover, it is now recognized that concomitant AMR may also occur and has been recognized as a cause of early unexplained graft loss, often with cholestasis. ${ }^{17}$ With modern immunosuppressive drugs, rejection is not considered as a significant cause of graft loss; however, in this cohort rejection, nonresponsive to standard treatment, appears to play a major role. AMR is a recognized cause of graft loss in other solid organ transplants, with the liver traditionally being thought as resistant to AMR; however, there is accumulating evidence that AMR may cause graft dysfunction in liver allografts. ${ }^{28-31}$

Cholestasis of varying degrees is a frequent histological finding in liver allograft biopsies and is seen in bile duct obstruction, acute and chronic rejection, preservationreperfusion injury, recurrent hepatitis $\mathrm{C}$, sepsis, and drug reactions. ${ }^{32,33}$ There are subtle differences between the histological features present in addition to the bilirubinostasis, which are associated with the clinical details often allow differentiation of the most likely cause.

In our series, histological findings of ACR overlapping with biliary features were the most common pattern found and have been the predominant driver for the management of these patients. Thus, despite the failure of antirejection treatment and the presence of histological findings that could not exclude a concomitant biliary process, the assessment of the biliary tree has been overlooked or severely delayed.

It is recognized that ACR may be seen in up to $32 \%$ of protocol biopsies, without biochemical evidence of graft dysfunction. ${ }^{34}$ In addition, the incidence of biliary complications after LT is reported to range from $5 \%$ to $25 \%,{ }^{35}$ increasing with risk factors, such as donor after circulatory death grafts, advanced donor age, prolonged warm ischemic time, graft steatosis, hepatic artery complications, and T tube use. ${ }^{36,37}$ Taken together, the presence of ACR on a biopsy, particularly if there is a failure to respond to treatment, should not be used as reassurance that there is not a biliary process occurring, and there should be an early trigger to investigate the biliary tree. From the findings of O'Leary, supported by some of our cases, the possibility of AMR should also be 
considered as contributing to the failure to respond to standard rejection treatment. Therefore, DSAs should be looked for and plasma exchange, rituximab, or other therapies for AMR considered. It is recognized that DSAs are associated with the development of biliary and vascular strictures, ${ }^{38}$ so the presence of a stricture does not exclude rejection as the underlying cause. Recently, an analysis of the incidence, costs, and outcomes of posttransplant biliary complications showed that the occurrence varies widely among transplant centers and that higher rates are a strong marker for increased risk of death, graft failure and healthcare spending. ${ }^{39}$

It is well known that macrovesicular steatosis greater than $30 \%$ is a predictor factor for reduced 1-year graft survival. ${ }^{19}$ In our cohort, donor-origin steatosis was found in 20 (59\%) of 34 postreperfusion liver biopsies; this was mild degree in 19 cases and moderate in 1 case. However, although graft steatosis might represent a risk factor contributing to persistent cholestasis, we believe that in our study population, the presence of mild steatosis did not significantly impact on graft outcomes. In contrast, the outcome of the patient who received the graft with moderate steatosis could have been influenced by the presence of the higher degree of steatosis, as a cofactor for persistent cholestasis.

This study is limited because of being a retrospective observational study, with a moderate number of recipients, limited radiological investigations to map out the biliary tree, interpretation of pathological findings, and recognition of AMR in the background where C4d and DSA tests are lacking. However, from our analysis, we believe that recipients with ACR, in whom biochemical cholestasis does not resolve after conventional antirejection treatment, should be investigated for an additional antibody mediated component to the rejection and would benefit from early investigation for biliary complications; this should be the case even if the US does not show biliary features. Concomitant biliary pathology, possibly secondary to rejection related injury to the peribiliary plexus, may lead to graft loss. ${ }^{40,41}$

In clinical practice, invasive assessment of the biliary tract by ERCP is still difficult in the first weeks after LT due to the risk of iatrogenic injury to "fresh" anastomosis, perforation, infection, bleeding, pancreatitis, and sedation-related complications. ${ }^{42-44}$ An alternative is MRCP, which has a sensitivity and specificity of $97 \%$ and $98 \%$, respectively, for diagnosing biliary obstruction in the nontransplant setting. ${ }^{45} \mathrm{~A}$ recent meta-analysis compared the accuracy of MRCP with ERCP in diagnosing biliary complications after $\mathrm{LT}^{46}$ : MRCP seems to have an excellent accuracy for detecting biliary obstruction after transplantation, but only if performed at centers with well-developed expertise in MRCP. ${ }^{47-49}$ Consequently, in our experience, the MRCP may be an appropriate test in patients with low to moderate suspicion of biliary obstruction, and its use could potentially avoid the unnecessary risks of ERCP in this clinical scenario (Figure 7).

Thus, in the last years, several authors proposed potential alternative approaches for the posttransplant cholestasis management. Recently, Lingala et $\mathrm{al}^{50}$ suggested using a hepatobiliary iminodiacetic acid scan and peak aspartate aminotransferase (AST) to identify which patients with post-LT cholestasis would benefit from ERCP, showing that a normal hepatobiliary iminodiacetic acid along with combination of peak AST and bilirubin levels offer a valuable tool in identifying patients who will not benefit from ERCP. Gilroy et $\mathrm{al}^{51}$ retrospectively analyzed all tests performed in LT recipients with biliary strictures and reported that

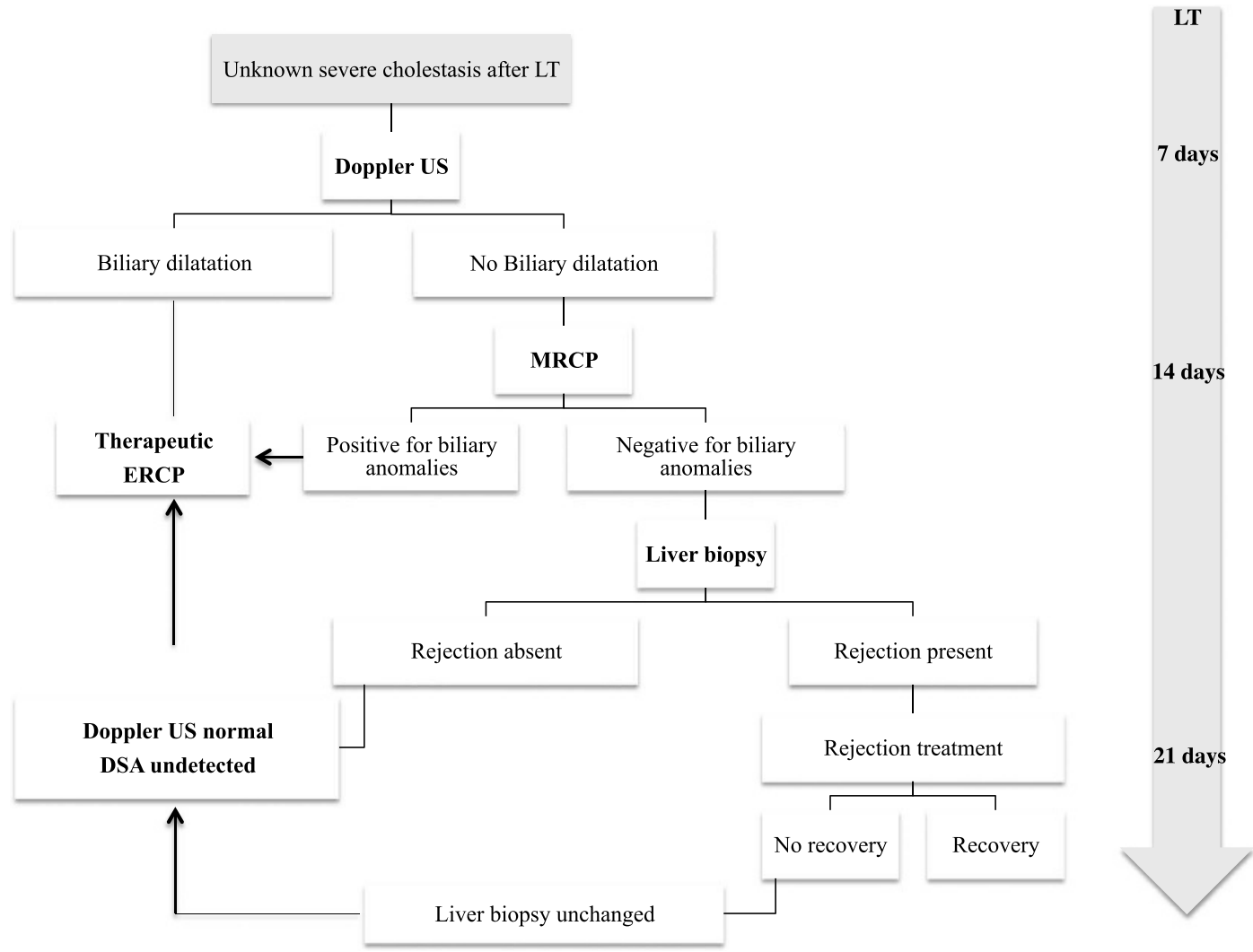

FIGURE 7. Proposed management of unresolved severe cholestasis after LT. 
US scanning is very insensitive for identifying biliary complications while liver histological findings of pericholangitis and lobular acute inflammation are strong predictors of biliary strictures. They suggest performing a graft biopsy before considering ERCP as diagnostic approach in patients without biliary dilatation on US scan. Moreover, a great interest in the relationship between the increased rate of biliary strictures/cholestasis after LT and the ischemic injury caused by low hepatic artery flow has recently been shown. ${ }^{52}$ This seems to be physiopathologically related not only to the ischemic bile duct damage but also to the functional impairment of bile secretion as previously described. ${ }^{53}$

In conclusion, unresolved severe cholestasis after transplantation influences patient/allograft outcome and its management is challenging. During the first weeks after LT, the role of US is unreliable in excluding early intrahepatic or extrahepatic biliary anomalies. Specific biliary radiological investigations are essential to rule out a biliary cause. Histologically, ACR in the presence of biliary features should trigger investigation of both biliary anastomotic complications and an AMR component, particularly, if there is no or limited response to standard rejection treatment. Close clinicopathological correlation and a multidisciplinary team comprising pathologists, hepatologists, and surgeons are necessary for the best outcome.

\section{REFERENCES}

1. Ben-Ari Z, Weiss-Schmilovitz H, Sulkes J, et al. Serum cholestasis markers as predictors of early outcome after liver transplantation. Clin Transplant. 2004;18:130-136.

2. Serenari M, Cescon M, Cucchetti $A$, et al. Liver function impairment in liver transplantation and after extended hepatectomy. World J Gastroenterol. 2013;19:7922-7929.

3. Banff Working Group; Demetris AJ, Adeyi O, Bellamy CO, et al. Liver biopsy interpretation for causes of late liver allograft dysfunction. Hepatology. 2006; 44:489-501.

4. O'Leary JG, Michelle Shiller S, Bellamy C, et al. Acute liver allograft antibody-mediated rejection: an inter-institutional study of significant histopathological features. Liver Transpl. 2014;20:1244-1255.

5. Kozlowski T, Rubinas T, Nickeleit V, et al. Liver allograft antibody-mediated rejection with demonstration of sinusoidal C4d staining and circulating donor-specific antibodies. Liver Transpl. 2011;17:357-368.

6. Watson R, Kozlowski T, Nickeleit V, et al. Isolated donor specific alloantibody-mediated rejection after $\mathrm{ABO}$ compatible liver transplantation. Am J Transplant. 2006;6:3022-3029.

7. Olthoff KM, Kulik L, Samstein B, et al. Validation of a current definition of early allograft dysfunction in liver transplant recipients and analysis of risk factors. Liver Transpl. 2010;16:943-949.

8. Deschênes M, Belle SH, Krom RA, et al. Early allograft dysfunction after liver transplantation: a definition and predictors of outcome. National Institute of Diabetes and Digestive and Kidney Diseases Liver Transplantation Database. Transplantation. 1998;66:302-310.

9. Deschenes M. Early allograft dysfunction: causes, recognition, and management. Liver Transpl. 2013;19:S6-S8.

10. Friedman $\mathrm{BH}$, Wolf $J H$, Wang $\mathrm{L}$, et al. Serum cytokine profiles associated with early allograft dysfunction in patients undergoing liver transplantation. Liver Transpl. 2012;18:166-176.

11. Strasberg SM, Howard TK, Molmenti EP, et al. Selecting the donor liver: risk factors for poor function after orthotopic liver transplantation. Hepatology. 1994;20:829-838.

12. González FX, Rimola A, Grande L, et al. Predictive factors of early postoperative graft function in human liver transplantation. Hepatology. 1994;20: 565-573.

13. Ali JM, Davies SE, Brais RJ, et al. Analysis of ischemia/reperfusion injury in time-zero biopsies predicts liver allograft outcomes. Liver Transpl. 2015; 21:487-499.

14. Feng S, Goodrich NP, Bragg-Gresham JL, et al. Characteristics associated with liver graft failure: the concept of a donor risk index. Am J Transplant. 2006;6:783-790.
15. Demetris AJ, Bellamy C, Hübscher SG, et al. 2016 Comprehensive Update of the Banff Working Group on Liver Allograft Pathology: introduction of antibody-mediated rejection. Am J Transplant. 2016;16:2816-2835.

16. Demetris A, Adams D, Bellamy C, et al. Update of the International Banff Schema for Liver Allograft Rejection: working recommendations for the histopathologic staging and reporting of chronic rejection. An International Panel. Hepatology. 2000;31:792-799.

17. O'Leary JG, Kaneku H, Demetris AJ, et al. Antibody-mediated rejection as a contributor to previously unexplained early liver allograft loss. Liver Transpl. 2014;20:218-227.

18. Demetris AJ, Zeevi A, O'Leary JG. ABO-compatible liver allograft antibody-mediated rejection: an update. Curr Opin Organ Transplant. 2015;20:314-324.

19. McCor mack L, Dutkowski P, El-Badry AM, et al. Liver transplantation using fatty livers: always feasible? J Hepatol. 2011;54:1055-1062.

20. Cutrin JC, Cantino D, Biasi F, et al. Reperfusion damage to the bile canaliculi in transplanted human liver. Hepatology. 1996:24:1053.

21. Moseley $\mathrm{RH}$, Wang W, Takeda $\mathrm{H}$, et al. Effect of endotoxin on bile acid transport in rat liver: a potential model for sepsis-associated cholestasis. Am J Physiol. 1996:271:G137-G146.

22. Mutimer D. CMV infection after liver transplantation. J Hepatol. 1996; 25:259.

23. Kadmon M, Klünemann C, Böhme M, et al. Inhibition by cyclosporin A of adenosine triphosphate-dependent transport from the hepatocyte into bile. Gastroenterology. 1993:104:1507-1514.

24. Martins FP, Kahaleh M, Ferrari AP. Management of liver transplantation biliary stricture: results from a tertiary hospital. World J Gastrointest Endosc. 2015;7:747-757

25. Barritt ASIV, Miller CB, Hayashi PH, et al. Effect of ERCP utilization and biliary complications on post-liver-transplantation mortality and graft survival. Dig Dis Sci. 2010;55:3602-3609.

26. Arain MA, Attam R, Freeman ML. Advances in endoscopic management of biliary tract complications after liver transplantation. Liver Transpl. 2013; 19:482-498.

27. Hubscher SG. Transplantation pathology. Semin Liver Dis. 2009;29:74.

28. O'Leary JG, Kaneku H, Susskind BM, et al. High MFI de novo MHC class II donor specific antibody formation impairs patient survival after liver transplantation. Am J Transplant. 2012;12(Suppl 3):68.

29. Del Bello A, Congy-Jolivet N, Danjoux M, et al. Donor-specific antibodies and liver transplantation. Hum Immunol. 2016;77:1063-1070.

30. Del Bello A, Congy-Jolivet N, Danjoux M, et al. De novo donor-specific anti-HLA antibodies mediated rejection in liver-transplant patients. Transp/ Int. 2015;28:1371-1382.

31. Demetris AJ, Bellamy CO, Gandhi CR, et al. Functional immune anatomy of the liver-as an allograft. Am J Transplant. 2016;16:1653-1680.

32. Demetris AJ. Transplantation Pathology of the liver. In: Odze RD, Goldblum JR, editors. Surgical Pathology of the GI Tract, Liver, Biliary Tract, and Pancreas. $2^{\text {nd }}$ ed. Philadelphia: Saunders/Elsevier; 2009 : 1169-1229.

33. Kukan M, Haddad PS. Role of hepatocytes and bile duct cells in preservation reperfusion injury of liver grafts. Liver Transpl. 2001;7:381-400.

34. Barlett AS, Ramadas R, Furness S, et al. The natural history of acute histologic rejection without biochemical graft dysfunction in orthotopic liver transplantation: a systematic review. Liver Transpl. 2002;8:1147.

35. Gastaca M. Biliary complications after orthotopic liver transplantation: a review of incidence and risk factors. Transplant Proc. 2012;44:1545-1549.

36. Taner CB, Bulatao IG, Willingham DL, et al. Events in procurement as risk factors for ischemic cholangiopathy in liver transplantation using donation after cardiac death donors. Liver Transpl. 2012;18:100-111.

37. Akamatsu N, Sugawara $Y$, Hashimoto D. Biliary reconstruction, its complications and management of biliary complications after adult liver transplantation: a systematic review of the incidence, risk factors and outcome. Transpl Int. 2011;24:379-392.

38. O'Leary JG, Demetris AJ, Friedman LS, et al. The role of donor-specific HLA alloantibodies in liver transplantation. Am J Transplant. 2014;14 779-787

39. Axelrod DA, Dzebisashvili N, Lentine KL, et al. Variation in biliary complication rates following liver transplantation: implications for cost and outcome. Am J Transplant. 2015;15:170-179.

40. Thethy S, Thomson BNj, Pleass H, et al. Management of biliary tract complications after orthotopic liver transplantation. Clin Transplant. 2004;18: 647-653

41. Thuluvath PJ, Pfau PR, Kimmey MB, et al. Biliary complications after liver transplantation: the role of endoscopy. Endoscopy. 2005;37:857-863.

42. Tse F, Barkun JS, Romagnuolo J, et al. Nonoperative imaging techniques in suspected biliary tract obstruction. HPB (Oxford). 2006;8:409-425. 
43. Jeurnink SM, Poley JW, Steyerberg EW, et al. ERCP as an outpatient treatment: a review. Gastrointest Endosc. 2008;68:118-123.

44. Freeman ML. Adverse outcomes of ERCP. Gastrointest Endosc. 2002;56: S273-S282.

45. Romagnuolo J, Bardou M, Rahme E, et al. Magnetic resonance cholangiopancreatography: a meta-analysis of test performance in suspected biliary disease. Ann Intern Med. 2003;139:547-557.

46. Jorgensen JE, Waljee AK, Volk ML, et al. Is MRCP equivalent to ERCP for diagnosing biliary obstruction in orthotopic liver transplant recipients? A meta-analysis. Gastrointest Endosc. 2011;73:955-962.

47. Kitazono MT, Qayyum A, Yeh BM, et al. Magnetic resonance cholangiography of biliary strictures after liver transplantation: a prospective doubleblind study. J Magn Reson Imaging. 2007;25:1168-1173.

48. Meersschaut V, Mortele KJ, Troisi R, et al. Value of MR cholangiography in the evaluation of postoperative biliary complications following orthotopic liver transplantation. Eur Radiol. 2000;10:1576-1581.
49. Beltran MM, Marugan RB, Oton E, et al. Accuracy of magnetic resonance cholangiography in the evaluation of late biliary complications after orthotopic liver transplantation. Transplant Proc. 2005; 37:3924-3925.

50. Lingala S, Satapathy S, Eason J, et al. Clinical algorithm to guide the need for ERCP for evaluation of early post liver transplant cholestasis. Transplantation. 2014;98:792.

51. Gilroy R, O'Neil M, Heronemus M, et al. Establishing a diagnostic algorithm for biliary strictures following liver transplantation. Transplantation. 2014;98:748.

52. Kim PT, Fernandez H, Gupta A, et al. Low measured hepatic artery flow increases rate of biliary strictures in deceased donor liver transplantation: an age-dependent phenomenon. Transplantation. 2017; 101:332-340.

53. Wagner M, Zollner G, Trauner M. Ischemia and cholestasis: more than (just) the bile ducts! Transplantation. 2008;85:1083-1085 